Bull. Korean Math. Soc. 50 (2013), No. 3, pp. 753-760

http://dx.doi.org/10.4134/BKMS.2013.50.3.753

\title{
REGULARITY OF SOLUTIONS OF 3D NAVIER-STOKES EQUATIONS IN A LIPSCHITZ DOMAIN FOR SMALL DATA
}

\author{
Hyo Suk Jeong, Namkwon Kim, And Minkyu Kwak
}

\begin{abstract}
We consider the global existence of strong solutions of the 3D incompressible Navier-Stokes equations in a bounded Lipschitz domain under Dirichlet boundary condition. We present by a very simple argument that a strong solution exists globally when the product of $L^{2}$ norms of the initial velocity and the gradient of the initial velocity and $L^{p, 2}, p \geq 4$ norm of the forcing function are small enough. Our condition is scale invariant and implies many typical known global existence results for small initial data including the sharp dependence of the bound on the volumn of the domain and viscosity. We also present a similar result in the whole domain with slightly stronger condition for the forcing.
\end{abstract}

\section{Introduction}

We consider the initial boundary value problem of the incompressible NavierStokes equations,

$$
\begin{array}{r}
u_{t}-\nu \Delta u+(u \cdot \nabla) u+\nabla p=f, \\
\nabla \cdot u=0,
\end{array}
$$

in a bounded domain $\Omega \subset \mathbb{R}^{3}$ with Lipschitz boundary $\partial \Omega$ or in $\mathbb{R}^{3}$ itself. Here $u$ denotes the velocity of a homogeneous, viscous incompressible fluid, $f$ is the density of force per unit volume, $p$ denotes the pressure, and $\nu$ is the kinematic viscosity. We require that the forcing function $f$ and the initial data $u_{0}$ satisfy

$$
\nabla \cdot f=\nabla \cdot u_{0}=0 .
$$

As a boundary condition, we use

$$
\left.u\right|_{\partial \Omega}=0
$$

when $\Omega$ is a bounded domain.

By the classical results of Leray and Hopf ([12], [18]), there exists a global weak solution of the Navier-Stokes equations if the initial data is in $L^{2}$ and

Received February 13, 2012.

2010 Mathematics Subject Classification. 35Q30, 35K15.

Key words and phrases. Navier-Stokes equations, global existence, strong solution.

The second author was partially supported by KRF 2010-0023456. The third author was supported in part by Korea Research Foundation (20110002446). 
satisfies (2). It is also known that the solution becomes necessarily strong (regular) for all regular data in two dimensional domain. But for the case $\Omega \subset \mathbb{R}^{3}$, global strong solutions have only been guaranteed for small initial data and small forcing term (see [2], [7], [8], [9], [13], [14], [23], [24], [25], [27] and references therein). The same is true for solutions in a negative Besov space (see [5], [22] and references therein) and for different boundary conditions (see [6], [10], [11], [15], [16], [17], [19], [21] and reference therein).

We here provide with some simple argument for global existence for small data in a Lipschitz domain, which recovers many known such results. Our argument also holds true in the whole $\mathbb{R}^{3}$. Concretely, we show the global existence of strong solutions if the initial data and the forcing function satisfy

$$
\left\|u_{0}\right\|^{2}\left\|\nabla u_{0}\right\|^{2}+4 \nu^{(4-4 p) / p} \lambda^{(4-3 p) / p}\|f\|_{p, 0}^{4} \leq \frac{\nu^{4}}{K^{4}}, \quad 4 \leq p \leq \infty
$$

for an interpolation constant $K$, which are independent of $|\Omega|$. Here, $|\Omega|$ denotes the volume of $\Omega, \lambda$ is the first eigenvalue of the Stokes operator, and

$$
\|f\|_{p, 0}^{p}=\int_{0}^{\infty}\|f\|_{L^{2}}^{p}(t) d t
$$

This condition is scale invariant under the Navier-Stokes scaling,

$$
v \rightarrow b v(a b t, a x), \quad \nu \rightarrow \frac{b}{a} \nu, \quad \lambda \rightarrow a^{2} \lambda, \quad f \rightarrow a b^{2} f(a b t, a x) .
$$

It implies that the $H^{1}$ norm of the initial velocity need not to be small for the global regularity. It also recovers that if the $L^{2}$ norm of the gradient of the initial velocity is small enough compared with a sixth power of the volume of $\Omega$, global existence is guaranteed when the forcing term vanishes.

This work has been done partially while the author is visiting the University of Minnesota for a sabbatical year and he is grateful to the School of Mathematics for their warm hospitalities and specially to Prof. G. R. Sell, Vladimir Sverak and Luan Hoang.

\section{Regularity}

We denote

$$
H=\left\{u \in L^{2}(\Omega) \mid \nabla \cdot u=0, u \cdot n(\partial \Omega, \cdot)=0\right\}
$$

when $\Omega$ is a bounded domain and

$$
H=\left\{u \in L^{2}\left(\mathbb{R}^{3}\right) \mid \nabla \cdot u=0\right\}
$$

when $\Omega=\mathbb{R}^{3}$. Also, $V \equiv H \cap W_{0}^{1,2}(\Omega)$ when $\Omega$ is bounded and $V \equiv H \cap W^{1,2}(\Omega)$ when $\Omega=\mathbb{R}^{3}$. We remark that if $\Omega$ is a bounded Lipschitz domain, $\|\nabla u\|$ is an equivalent norm for $V$ due to the Poincaré type inequality. For convenience's sake, we also denote $\|\cdot\|_{p}=\|\cdot\|_{L^{p}},\|\cdot\|_{2}=\|\cdot\| \cdot \mathbb{P}$ is the Leray projection 
of $L^{2}(\Omega)=L^{2}\left(\Omega, \mathbb{R}^{3}\right)$ into $H$. Now, applying the Leray projection, we can reformulate (1) in the following evolutionary equation on the Hilbert space $V$,

$$
u_{t}+\nu A u+B(u, u)=\mathbb{P} f,
$$

where $A u=-\mathbb{P} \triangle u$, and the bilinear form $B(u, v)=\mathbb{P}(u \cdot \nabla) v$. We will be interested in solutions of (3) with the initial data $u_{0}$ and $f=f(t)$ satisfying

$$
u_{0} \in V, \quad f(t) \in L^{\infty}([0, \infty), H) .
$$

Theorem 2.1. Let $\Omega$ be a bounded Lipschitz domain in $\mathbb{R}^{3}$. The Navier-Stokes evolutionary equation (3) has a solution

$$
u \in C^{0}([0, \infty), H) \cap L^{\infty}((0, \infty), V)
$$

whenever $u_{0}$ and $f$ satisfy

$$
\left\|u_{0}\right\|^{2}\left\|\nabla u_{0}\right\|^{2}+4 \nu^{\frac{4-4 p}{p}} \lambda^{\frac{4-3 p}{p}}\|f\|_{p, 0}^{4} \leq \frac{\nu^{4}}{K^{4}}
$$

for some $p \in[4, \infty]$. Here, $K$ is a scale invariant embedding constant, $\lambda$ is the first eigenvalue of the Stokes operator, and $\|\cdot\|_{p, 0}=\|\cdot\|_{L^{p}\left((0, \infty), L^{2}\right)} \cdot$ Moreover, in this case

$$
\|\nabla u\|^{2}(t) \leq\left\|\nabla u_{0}\right\|^{2}+4 \nu^{\frac{2-2 p}{p}} \lambda^{\frac{2-p}{p}}\|f\|_{p, 0}^{2}
$$

for all $t>0$.

Proof. Since $u_{0} \in H^{1}$ and $f \in L^{2}\left(0, T ; L^{2}\right)$ for all $T>0$, there exists a local in time unique strong solution, $u$ corresponding to $u_{0}$ and $f$. Suppose that this solution blows up in a finite time. This means that there exists a maximal time of existence, $T^{*}>0$ at which the solution quits to belong $H^{1}$. Now, let us restrict ourself $t<T^{*}$. By taking the scalar product of (3) with $u$ and using the fact that $\langle B(u, u), u\rangle=0$, we find that

$$
\frac{d}{d t}\|u\|^{2}+2 \nu\|\nabla u\|^{2} \leq 2\|f\|\|u\|
$$

Now, we borrow the following inequality for a bounded Lipschitz domain from [3] and [1]

$$
\|u\|_{\infty} \leq K\|\nabla u\|^{1 / 2}\|A u\|^{1 / 2}
$$

to get

$$
\begin{aligned}
|\langle B(u, u), A u\rangle| & =\left|\int u \cdot \nabla u \cdot A u\right| \\
& \leq\|u\|_{L^{\infty}}\|\nabla u\|\|A u\| \\
& \leq K\|\nabla u\|^{3 / 2}\|A u\|^{3 / 2} \\
& \leq K\|u\|^{1 / 2}\|\nabla u\|^{1 / 2}\|A u\|^{2} .
\end{aligned}
$$

Here, in the last line, we use the inequality

$$
\|\nabla u\|^{2}=-\int u \cdot A u \leq\|u\|\|A u\| .
$$


Note that $K$ is a scaling invariant constant which may depend on the Lipschitz norm of $\partial \Omega$. Taking the scalar product of (3) with $A u$ and using the above estimate, we obtain

$$
\frac{d}{d t}\|\nabla u\|^{2}+2 \nu\|A u\|^{2} \leq 2\|f\|\|A u\|+K(\|u\|\|\nabla u\|)^{1 / 2}\|A u\|^{2} .
$$

Next, we multiply (7) by $\|\nabla u\|^{2}$ and (10) by $\|u\|^{2}$ and adding them to have

$$
\begin{aligned}
& \frac{d}{d t}\left(\|u\|^{2}\|\nabla u\|^{2}\right)+2 \nu\|\nabla u\|^{4}+2 \nu\|u\|^{2}\|A u\|^{2} \\
\leq & 2\|f\|\|u\|\left(\|\nabla u\|^{2}+\|u\|\|A u\|\right)+K(\|u\|\|\nabla u\|)^{1 / 2}\|u\|^{2}\|A u\|^{2} .
\end{aligned}
$$

By the Hölder inequality and (9), we have

$$
\begin{aligned}
2\|f\|\|u\|\left(\|\nabla u\|^{2}+\|u\|\|A u\|\right) & \leq 4\|f\|\|u\|^{2}\|A u\| \\
& \leq \nu\|u\|^{2}\|A u\|^{2}+\nu\|\nabla u\|^{4}+\frac{4}{\nu^{3} \lambda^{2}}\|f\|^{4} .
\end{aligned}
$$

Denoting $G=\|u\|^{2}\|\nabla u\|^{2}$, we thus arrive at the differential inequality

$$
\frac{d}{d t} G+\nu \lambda G \leq\left[K G^{1 / 4}-\nu\right]\|u\|^{2}\|A u\|^{2}+\frac{4}{\nu^{3} \lambda^{2}}\|f\|^{4} .
$$

By (5), $K G(0)^{1 / 4}-\nu \leq 0$. And, since $G(t)$ is continuous for $t<T^{*}$, there exists a maximal $T_{1} \leq T^{*}$ such that $K G^{1 / 4}-\nu \leq 0$ on $\left[0, T_{1}\right)$. Then we have by the Grönwall inequality

$$
\begin{aligned}
G(t) & \leq G(0) e^{-\nu \lambda t}+\frac{4}{\nu^{3} \lambda^{2}} \int_{0}^{t}\|f\|^{4} e^{\nu \lambda(s-t)} d s \\
& <G(0)+4 \nu^{(4-4 p) / p} \lambda^{(4-3 p) / p}\|f\|_{p, 0}^{4}, \quad 4 \leq p \leq \infty
\end{aligned}
$$

for $t<T_{1}$ (Equality cannot hold in the last line). This implies that $K G(t)^{1 / 4}-$ $\nu<0$ for all $t<T_{1}$ by (5) and thus $T_{1}=T^{*}$. Now, using $G(t) \leq \frac{\nu^{4}}{K^{4}}$ for $t<T^{*}$, we have from $(10)$

$$
\frac{d}{d t}\|\nabla u\|^{2}+\frac{\nu}{2}\|A u\|^{2} \leq \frac{2}{\nu}\|f\|^{2}, \quad t<T^{*} .
$$

By the Grönwall inequality and the inequality

$$
\lambda\|\nabla u\|^{2} \leq\|A u\|^{2},
$$

we have finally

$$
\begin{aligned}
\|\nabla u\|^{2}(t) & \leq\|\nabla u\|^{2}(0) e^{-\frac{\nu \lambda}{2} t}+\frac{2}{\nu} \int_{0}^{t}\|f\|^{2} e^{\frac{\nu \lambda}{2}(s-t)} d s \\
& \leq\|\nabla u\|^{2}(0)+4 \nu^{\frac{2-2 p}{p}} \lambda^{\frac{2-p}{p}}\|f\|_{p, 0}^{2}
\end{aligned}
$$

for all $t<T^{*}$. This leads a contradiction and we conclude $T^{*}=+\infty$. The above inequality shows (6) then for all $t>0$. 
Remark. 1. The condition (5) is in a sense a condition of smallness of the initial data and external force. However, this condition allows for initial data with large $H^{1}$ norm provided that the $L^{2}$ norm of the initial data is small enough. In particular, when $f=0$, the above theorem tells that even for initial data with large $H^{1}$ norm, there exists a globally regular solution if $\left\|u_{0}\right\|$ is small enough compared with $\nu^{2}\left\|\nabla u_{0}\right\|^{-1}$.

2. Although we show the above theorem under Dirichlet boundary condition for a bounded domain, it still holds for a cylindrical domain under mixed no-slip and periodic boundary condition.

3. The condition $p \geq 4$ could be relaxed to $p \geq 2$ if we just want to obtain the global regularity of the solution. However, we may lose the simple scale invariant bound of $H^{1}$ norm of the solution in this approach.

The above theorem is also in consistent with the previously known global regularity result for small initial data. For example, the case $p=2$ and $\Omega$ being $C^{2}$ is reduced to Theorems 9.3 and 9.4 in [7]. It also gives the dependence of the bound on the volumn of the domain. The following corollary reveals this, which is a consequence of a simple embedding theorem.

Corollary 2.2. Given initial data $u_{0} \in H^{1} \cap H$ and $4 \leq p \leq \infty$. There exists a scaling invariant constant $C$ such that if

$$
\left\|\nabla u_{0}\right\| \leq C \nu|\Omega|^{-1 / 6}, \quad\|f\|_{p, 0} \leq C \nu^{(2 p-1) / p}|\Omega|^{-(3 p-4) / 6 p},
$$

there exists a globally regular solution of (3).

Proof. Since $\lambda\left\|u_{0}\right\|^{2} \leq\left\|\nabla u_{0}\right\|^{2}$, the condition (5) is satisfied if

$$
\left\|\nabla u_{0}\right\|^{4} \leq \frac{\nu^{4}}{5 K^{4}} \lambda, \quad\|f\|_{p, 0}^{4} \leq \frac{\nu^{(8 p-4) / p} \lambda^{(3 p-4) / p}}{5 K^{4}} .
$$

Meanwhile, since

$$
\lambda=\inf _{v \in D\left(A^{1 / 2}\right)} \frac{\|\nabla v\|^{2}}{\|v\|^{2}},
$$

the scaling $x \rightarrow|\Omega|^{1 / 3} x$ gives $\lambda(\Omega)=|\Omega|^{-2 / 3} \lambda\left(\Omega_{0}\right)$. Here, $\Omega_{0}$ is a scaling of $\Omega$ with unit volume. Further, $\lambda\left(\Omega_{0}\right) \geq \lambda^{D}\left(\Omega_{0}\right)$, the first Dirichlet eigenvalue by the above definition. Thus, denoting the ball of unit volume by $B, \lambda\left(\Omega_{0}\right) \geq$ $\lambda^{D}(B)$ by symmetrization. Putting these together and defining $C$ suitably, we finish the proof.

The above corollary in particular tells that when $p=2$, both of the bounds grows like $|\Omega|^{-1 / 6}$.

Under slightly stronger condition on the external force, an analogy of the above theorem holds on the whole of $\mathbb{R}^{3}$.

Theorem 2.3. When $\Omega=\mathbb{R}^{3}$, (3) has a solution

$$
u \in C^{0}([0, \infty), H) \cap L^{\infty}((0, \infty), V)
$$


whenever $u_{0}$ and $f$ satisfy

$$
\left\|u_{0}\right\|^{2}\left\|\nabla u_{0}\right\|^{2}+\frac{2}{\nu}\left(\left\|u_{0}\right\|+\int_{0}^{\infty}\|f\|\right)^{2} \int_{0}^{\infty}\|f\|^{2} \leq \frac{\nu^{4}}{K} .
$$

Further,

$$
\|\nabla u\|^{2}(t) \leq\left\|\nabla u_{0}\right\|^{2}+\frac{1}{\nu} \int_{0}^{t}\|f\|^{2}
$$

for all $t>0$.

Proof. Exactly by the same argument as in the previous theorem, (7) and (10) holds in this case, too. In particular, the inequality (8) reduces to the Gargliardo-Nirenberg inequality in this case and is well-known. Dividing by $2\|u\|$, we reduce $(7)$ further to

$$
\frac{d}{d t}\|u\| \leq\|f\|
$$

Integrating the above inequality, we have

$$
\|u\|(t) \leq\left\|u_{0}\right\|+\int_{0}^{t}\|f\| .
$$

Meanwhile, from (7) and (10), we again have (11). To manipulate (11) further, we estimate the forcing term as follows.

$$
2\|f\|\|u\|\left(\|\nabla u\|^{2}+\|u\|\|A u\|\right) \leq \nu\|\nabla u\|^{4}+\nu\|u\|^{2}\|A u\|^{2}+\frac{2}{\nu}\|f\|^{2}\|u\|^{2} .
$$

Then, (11) is reduced to

$$
\frac{d}{d t} G \leq\left[K G^{1 / 4}-\nu\right]\|u\|^{2}\|A u\|^{2}+\frac{2}{\nu}\|f\|^{2}\|u\|^{2} .
$$

Here, $G=\|u\|^{2}\|\nabla u\|^{2}$ as before. Again by the continuation argument and the Grönwall inequality, $G(t) \leq G(0)$ if

$$
G(0)+\frac{2}{\nu} \int_{0}^{\infty}\|f\|^{2}\|u\|^{2} \leq \frac{\nu^{4}}{K^{4}}
$$

By (14), the above inequality is satisfied under (12). Finally, once $G(t) \leq$ $G(0) \leq(\nu / K)^{4}$, from $(10)$,

$$
\begin{aligned}
\frac{d}{d t}\|\nabla u\|^{2}+\nu\|A u\|^{2} & \leq\|f\|\|A u\| \\
& \leq \frac{1}{\nu}\|f\|^{2}+\nu\|A u\|^{2} .
\end{aligned}
$$

Integrating the above inequality, we recover (13) and finish the proof. 


\section{References}

1] R. A. Adams, Sobolev Spaces, Academic Press, New York, 1975.

[2] J. D. Avrin, Large-eigenvalue global existence and regularity results for the NavierStokes equations, J. Differential Equations 127 (1996), no.2, 365-390.

[3] R. Brown and Z. Shen, Estimates for the Stokes operator in Lipschitz domains, Indiana Univ. Math. J. 44 (1995), no. 4, 1183-1206.

[4] H. J. Choe and K. Hideo, The Stokes problem for Lipschitz domains, Indiana Univ. Math. J. 51 (2002), no. 5, 1235-1259.

[5] J. Y. Chemin, I. Gallagher, and M. Paicu, Global regularity for some classes of large solutions to the Navier-Stokes equations, Ann. of Math. (2) 173 (2011), no. 2, 983-1012.

[6] I. Chueshov, G. Raugel, and A. M. Rekalo, Interface boundary value problem for the Navier-Stokes equations in thin two-layer domains, J. Differential Equations 208 (2005), no. 2, 449-493.

[7] P. Constantin and C. Foias, Navier-Stokes Equations, University of Chicago Press, Chicago, 1988.

[8] P. Deuring and W. von Wahl, Strong solutions of the Navier-Stokes system in Lipschitz bounded domains, Math. Nachr. 171 (1995), 111-148.

[9] H. Fujita and T. Kato, On the Navier-Stokes initial value problem, Arch. Rational Mech. Anal. 16 (1964), 269-315.

[10] L. T. Hoang, Incompressible fluids in thin domains with Navier friction boundary conditions (I), J. Math. Fluid Mech. 12 (2010), no. 3, 435-472.

[11] L. T. Hoang and G. R. Sell, Navier-Stokes equations with Navier boundary conditions for an oceanic model, J. Dynam. Differential Equations 22 (2010), no. 3, 563-616.

[12] E. Hopf, Über die Anfangswertaufgabe fur die hydrodynamischen Grudgleichungen, Math. Nachr. 4 (1951), 213-231.

[13] D. Iftimie, The 3D Navier-Stokes equations seen as a perturbation of the 2D NavierStokes equations, Bull. Soc. Math. France 127 (1999), no. 4, 473-517.

[14] D. Iftimie and G. Raugel, Some results on the Navier-Stokes equations in thin 3D domains, J. Differential Equations 169 (2001), no. 2, 281-331.

[15] D. Iftimie, G. Raugel, and G. R. Sell, Navier-Stokes equations in thin $3 D$ domains with Navier boundary conditions, Indiana Univ. Math. J. 56 (2007), no. 3, 1083-1156.

[16] M. Kwak and N. Kim, Global existence for 3D Navier-Stokes equations in a thin periodic domain, J. Korean Soc. Ind. Appl. Math. 15 (2011), no. 2, 143-150.

[17] I. Kukavica and M. Ziane, Regularity of the Navier-Stokes equation in a thin periodic domain with large data, Discrete Contin. Dyn. Syst. 16 (2006), no. 1, 67-86.

[18] J. Leray, Sur le mouvement d'un liquide visqueux emplissant l'espace, Acta Math. 63 (1934), no. 1, 193-248.

[19] M. Mitrea and S. Monniaux, The nonlinear Hodge-Navier-Stokes equations in Lipschitz domains, Differential Integral Equations 22 (2009), no. 3-4, 339-356.

[20] S. Monniaux, On uniqueness for the Navier-Stokes system in 3D-bounded Lipschitz domains, J. Funct. Anal. 195 (2002), no. 1, 1-11.

[21] S. Montgomery-Smith, Global regularity of the Navier-Stokes equations on thin three dimensional domains with periodic boundary conditions, Electron. J. Differential Equations 1999 (1999), no. 19, 1-19.

[22] M. Paicu and Z. Zhang, Global regularity for the Navier-Stokes equations with some classes of large initial data, Anal. PDE 4 (2011), no. 1, 95-113.

[23] G. Raugel and G. R. Sell, Navier-Stokes equations on thin 3D domains. I. Global attractors and global regularity of solutions, J. Amer. Math. Soc. 6 (1993), no. 3, 503-568.

[24] G. R. Sell and Y. You, Dynamics of Evolutionary Equations, Applied Math. Sciences 143, Springer, Berlin, 2002. 
[25] R. Temam, Navier-Stokes Equations and Nonlinear Functional Analysis, CBMS Regional Conference Series, No. 66, SIAM, Philadelphia, 1995.

[26] R. Temam and M. Ziane, Navier-Stokes equations in three-dimensional thin domains with various boundary conditions, Adv. Differential Equations 1 (1996), no. 4, 499-546.

[27] K. Wang, On global regularity of incompressible Navier-Stokes equations in $\mathbb{R}^{3}$, Commun. Pure Appl. Anal. 8 (2009), no. 3, 1067-1072.

Hyo Suk JeOng

Department of Mathematics

Chonnam National University

KWANGJU 500-757, Korea

E-mail address: yozosukyoo@hanmail.net

NAMKWON KIM

Department of Mathematics

Chosun University

KWANGJU 501-759, Korea

E-mail address: kimnamkw@chosun.ac.kr

MINKYU KWAK

Department of Mathematics

Chonnam National University

KwangJu 500-757, Korea

E-mail address: mkkwak@jnu.ac.kr 